\title{
EVALUATION OF CHRONIC HEADACHE BY COMPUTED TOMOGRAPHY: A RETROSPECTIVE STUDY
}

\author{
Anish Subedee
}

\begin{abstract}
:
Objective: To find out the proportion of intracranial abnormalities in patients with chronic headache without neurologic abnormality with the use of computed tomography (CT) and to compare the results with similar studies done previously.

Materials and methods: CT images of 56 patients with chronic/recurrent headache and normal neurological findings were reviewed retrospectively. In 38 of 56 patients, both plain and contrast enhanced CT were done. Patients were divided into three groups according to the CT findings: those with no abnormality, those with minor abnormality (that did not alter patient management) and those with clinically significant abnormality. Proportion of patients in each group was found out and results were compared with previous studies with similar study design. $Z$ test was used to evaluate whether the difference in proportions of patients in our study and previous study was statistically significant or not.
\end{abstract}

Results: Of the 56 patients, 50 had normal CT (89.28\%), four had minor abnormality (7.14\%) that did not alter patient management and two had significant lesions (3.57\%). Contrast enhanced CT did not improve lesion detection. The minor findings detected were sub-ependymal calcifications of Tuberous sclerosis, calcified neurocysticercosis and old lacunar infarctions in external capsule. Clinically significant lesions detected were small ring enhancing lesion (neurocysticercosis or tuberculoma) and pineal cyst. Results of this study were compared with previous study with similar study design. The $Z$ test showed that the difference in proportions in these studies was not statistically significant ( $p=0.0708$ for minor findings and $p=0.2033$ for significant findings).

Conclusion: The proportion of intracranial abnormalities detected by CT in this study was similar to that of previous studies. The use of intravenous contrast material administration did not improve its yield. This corroborates the evidence that the ability of CT scan in detecting significant intracranial pathology is poor in patients with chronic headache without neurologic abnormality.

Key words: Chronic headache, CT

\section{Introduction:}

Headache is an almost universal experience and one of the most common symptoms in medical practice. It varies from an infrequent and trivial nuisance to a pointer to serious disease (1). Population based estimates suggest that about $4 \%$ of adults have daily or near daily headache (2). Although majority of the patients who present with chronic or recurrent headache have no neurologic abnormality, many patients undergo evaluation with computed tomography (CT) and more recently, 


\section{Original Article}

magnetic resonance imaging (MRI) to exclude important abnormalities (3).

The studies that have been done so far show that CT is of extremely low yield in patients who undergo imaging for chronic headache without neurologic abnormality (4-6). MR imaging, which is more sensitive than CT in detecting intracranial abnormality (7), has also been found to be unrewarding in evaluation of chronic or recurrent headache without neurological abnormality (3).Yet patient's demand for thorough and high-tech evaluation coupled with the low threshold among doctors for requesting these investigations has accelerated the use of CT and MRI despite the evidences against their use.

Due to unavailability and higher cost of MRI, CT is requested more frequently in our set up. However no data exist in our country about the use of CT for evaluation of chronic headache and normal neurological finding. Thus, the purpose of this study was to evaluate the ability of $\mathrm{CT}$ in evaluation of chronic headache without neurologic abnormality. Specifically, this study aimed to find out the proportion of intracranial abnormalities in this group of patient and compare the results with similar studies done previously.

\section{Materials and methods:}

Setting: The study was carried out at department of radiology in Chitwan Medical College Teaching Hospital, Bharatpur, Nepal.

- The clinical data and the CT images of the patients undergoing cranial CT scan for the evaluation of chronic headache were retrospectively reviewed.

\section{Chronic Headache and CT Scan}

- Clinical information was collected from the requisition form supplied by the referring doctor. CT images were reviewed from the digital archive of the department.

- All the patients with the chief complaint of chronic / recurrent / long standing headache were included in the study. Exclusion criteria were as follows:

- Inadequately filled requisition forms

- Presence of other neurologic symptoms like trauma, seizures.

- Abnormal neurological finding in clinical examination.

- Patients with diagnosed CNS abnormality

- Of the consecutive 515 head CT scans done between 2009-04-28 and 2009-10-01, only 56 scans were included in the study.

CT scan: All the patients underwent scan with same 16 slice CT scanner (Bright speed, GE). Helical scan of the cranium from base of the skull to the vertex was done with collimation of $5 \mathrm{~mm}$ and multi-planar reconstruction at $0.6 \mathrm{~mm}$ thickness when desirable. Although no definite departmental protocols were present for indication of intravenous contrast material administration, both plain and contrast enhanced scans were done in 38 patients when referring doctor or radiologist recommended its use.

All the images were interpreted by the same radiologist and the imaging results were divided into three groups:

i) those with no abnormality (normal scans)

ii) those with minor abnormality (that did not alter patient management)

iii) those with clinically significant abnormality (which may result in chronic/recurrent headache) 


\section{Original Article}

\section{Results:}

Of the total $789 \mathrm{CT}$ scans done during the study period, 515 scans $(65.27 \%)$ were that of head and only 56 patients were included in the study. Of the 56 patients, 16 were male and 40 were female and the age range was 5-72 years.

Of the 56 patients, 50 had normal CT (89.28 $\%), 4$ had minor abnormality (7.14\%) that did not alter patient management and 2 had significant lesions $(3.57 \%)$. Of the patients with minor findings, one had sub-ependymal calcifications of Tuberous Sclerosis, two had calcified neurocysticercosis and one had old lacunar infarctions in $b / 1$ external capsule. Two minor findings were seen in male and two in female group. Of the significant findings detected, one was a small ring enhancing lesion (either neurocysticercosis or tuberculoma) in a 12 years girl and another was a pineal cyst measuring $14 \mathrm{~mm}$ in diameter in a 30 years female.

The results of present study were compared with previous study with similar study design using CT for evaluation of headache.

\section{Chronic Headache and CT Scan}

Dumas MD et al (6) had found that of the 402 CT scans $14(3.48 \%)$ revealed minor findings and $4(0.99 \%)$ revealed significant findings. $Z$ test was used to evaluate whether the difference in proportions of patients in our study and the study of Dumas MD et al was statistically significant or not. For minor findings, the $Z$ value was $1.47(p=0.0708)$ and for significant findings $Z$ value was 0.83 $(p=0.2033)$. The difference in proportions in these studies was not significant.

Age and sex wise distribution of the disease was not evaluated for statistical significance because of very small number of observations in individual group.

Among the 56 patients, 38 patients had been investigated with both plain and contrast enhanced CT scans. Of these, none of patients showed additional advantage of contrast enhanced over plain CT. Statistical significance was not evaluated because of small number of patients and number of observations in one of the column being zero.

Table 1: Age distribution:

\begin{tabular}{|c|c|}
\hline Age & No. of patients \\
\hline$<20$ & 11 \\
\hline $20-39$ & 29 \\
\hline $40-59$ & 13 \\
\hline$\geq 60$ & 3 \\
\hline
\end{tabular}




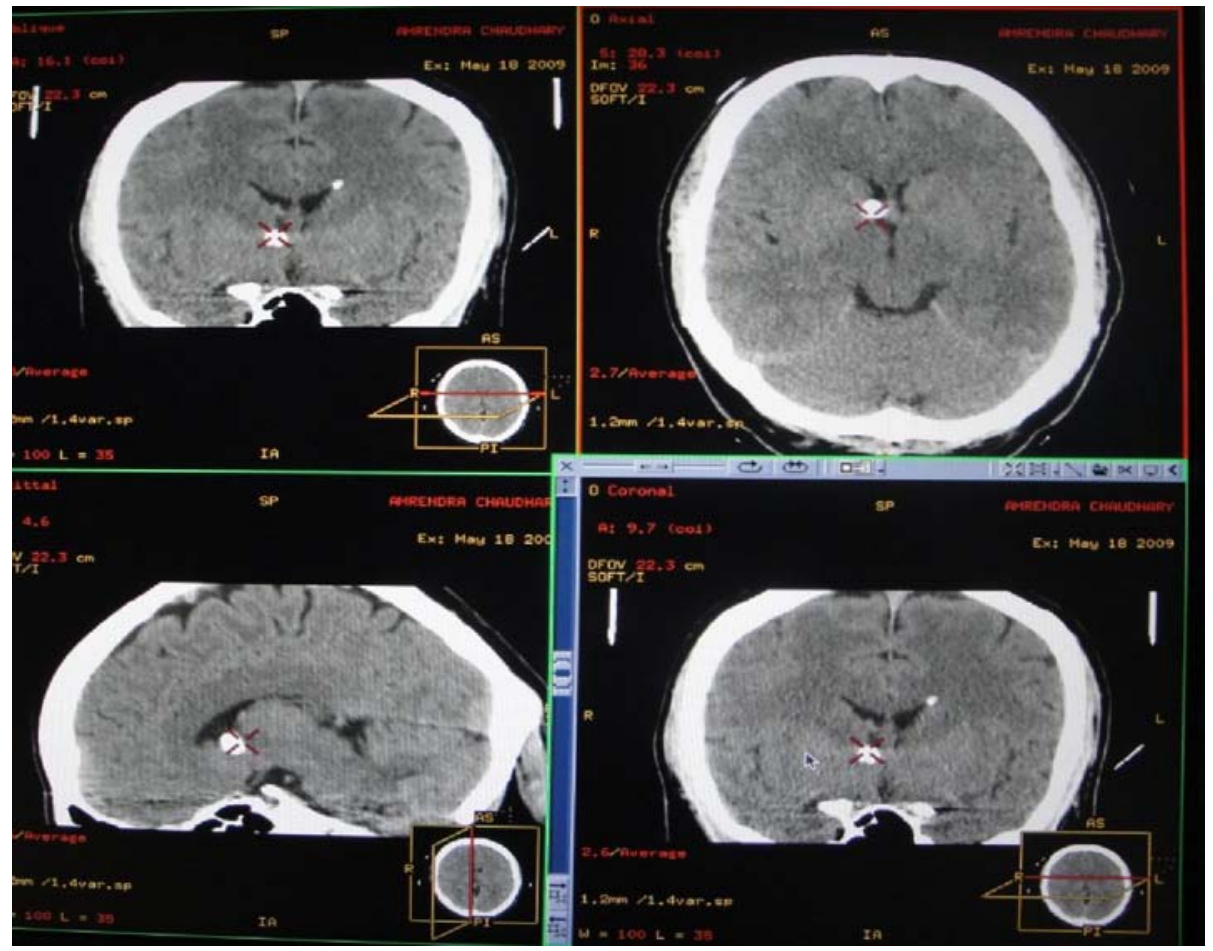

Fig 1: Sub-ependymal calcifications in Tuberous Sclerosis
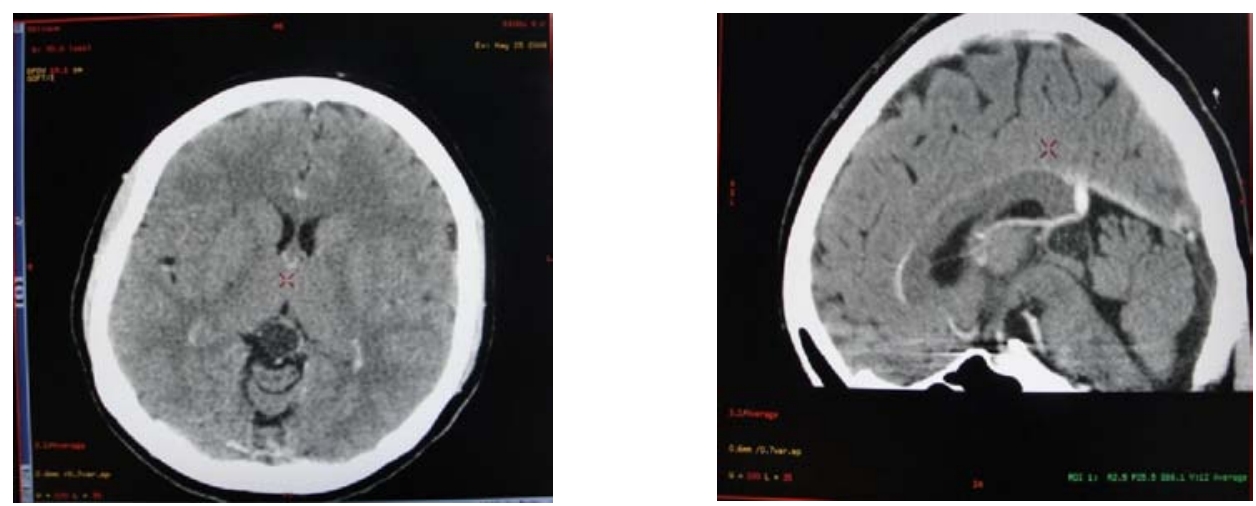

Fig 2: Pineal cyst

\section{Discussion:}

In evaluation of chronic headache without neurological abnormality, balancing patient's concern and available evidences regarding use of neuroimaging has been a tight rope walk for clinicians. In practice however tendency towards liberal use of imaging is prevalent. American Academy of
Neurology in 2000 had published practice guidelines for imaging in headache (8). Neuroimaging recommendations for nonacute headache are as follows:

Consider neuroimaging in:

- Patients with an unexplained abnormal finding on the neurologic examination (Grade B) 
- Patients with atypical headache features or headaches that do not fulfill the strict definition of migraine or other primary headache disorder (or have some additional risk factor, such as immune deficiency), when a lower threshold for neuroimaging may be applied (Grade C)

- Neuroimaging is not usually warranted in patients with migraine and a normal neurologic examination (Grade B).

- No evidence-based recommendations are established for the following:

- Presence or absence of neurologic symptoms (Grade C) Tension-type headache (Grade C)

The US headache consortium (9) later said that the evidences were insufficient to define the role of neuroimaging in evaluation of chronic or recurrent headaches without neurological abnormality. However it made similar recommendations as that of American Academy of Neurology and further recommended larger study involving more than 1000 patients to settle the issue.

Various studies have been done to evaluate the ability of neuro-imaging in detecting abnormalities in patients with chronic or recurrent headache without neurological abnormality. Definition of minor and significant abnormality however was not uniform among the studies performed earlier. Only two studies were found having study design similar to our study, one using $\mathrm{CT}$ and another using MR imaging for evaluation of headache. Hence the results of present study were compared only with the study of Dumas et al who had used CT for evaluation of headache.

The present study found that $7.14 \%$ of patients had minor abnormality and 3.57\% had significant abnormality mandating change in line of management. Presence of pineal cyst was taken as significant on the basis of a study conducted at Germany (10) in 51 patients with pineal cysts which showed significant causal relationship between pineal cyst and headache. Comparison of proportions of patients with minor and significant abnormality with prior study showed no significant statistical difference (6).

Other studies also showed results similar to our study. According to a meta-analysis (9), significant clinical abnormalities detected in CT scans in patients with unspecified headache ranged from $0.0 \%$ to $6.7 \%$ in ten studies. Though MR imaging is considered more sensitive than $\mathrm{CT}$, one study done in Japan concluded that MR is an unrewarding technique in evaluation of patients with chronic or recurrent headache and neurologic findings (3).

Value of intravenous contrast material administration in this group of patient has also been evaluated previously by different authors $(11,12)$. None of the studies have shown value of contrast material administration in this group of patient which is similar to our findings. Moreover contrast administration means additional cost to the patient along with the risk of adverse drug reactions as well.

Some of the previous studies have tried to evaluate the cost effectiveness of CT scan in this group of patient $(6,13)$. Both show very high cost per single significant finding. CT scan is one of the expensive investigations in our country also. Its use in evaluation of a 


\section{Original Article}

problem which is universal in magnitude especially when the diagnostic yield is so little should be seriously re-evaluated in underdeveloped country like Nepal.

Many patients seek attention for their headache because of the anxiety that they are having significant intracranial disease. It has been argued that the relief patient feels that he/she does not have any significant intracranial disease is a worthwhile outcome. However anxiety relief is subjective and difficult to measure. Furthermore, patients prone to anxiety may not feel completely relieved even after undergoing CT scan.

There were few important limitations of this study. First, lack of records of neurological evaluation was an important limitation which might have misled us in patient selection. Our study being retrospective in nature, and most of the patients coming from out patient department, record of complete neurological evaluation could not be obtained. Second, small number of patients in the study was another limitation. The number of patients in individual sub group, in which patients were categorized according to their imaging findings, was subsequently very small.

\section{Conclusion:}

In the patients with chronic/recurrent headache without neurologic abnormality, CT detected minor abnormalities in 7.14\% and significant abnormalities in $3.57 \%$ of patients. The use of intravenous contrast material administration did not improve its yield. This result is consistent with findings of previous studies. This further corroborates the evidence that the ability of CT scan in detecting significant intracranial pathology is poor in this group of patients. However large studies need to be done to
Chronic Headache and CT Scan

establish the role of CT scan in evaluation of the patients with chronic headache.

\section{References:}

1. Clarke CRA. Neurological Disease. In: Kumar $\mathrm{P}$, Clark M, editors. Clinical Medicine. Sixth ed. Elsevier Limited; 2005. p. 1174.

2. Goadsby PJ, Raskin NH. Headache. In: Fauci AS, Braunwald EB, Casper DL et al, editors. Harrison's Principles of Internal Medicine. $17^{\text {th }}$ ed. McGraw Hill Medical; 2008. p. 103.

3. Tsushima Y, Endo K. MR imaging in the evaluation of chronic or recurrent headache. Radiology 2005; 235:575-579.

4. Practice parameter: the utility of neuroimaging in the evaluation of headache in patients with normal neurological examinations (summary statement). Report of the Quality Standards Subcommittee of the American Academy of Neurology. Neurology 1994; 44:1353-1354.

5. Frishberg BM. The utility of neuroimaging in the evaluation of headache in patients with normal neurologic examinations. Neurology 1994; 44: 1191-1197.

6. Dumas MD, Pexman JH, Kreeft JH. Computed tomography evaluation of patients with chronic headache. [pulished correction appears in Can Med Assoc J. 1995; 152:158]. CMAJ 1994; 151:1447-1452.

7. Haughton VM, Rimm AA, Sobocinski KA, et al. A blinded clinical comparison of MR imaging and CT in neuroradiology. Radiology1986; 160:751-755.

8. Practice parameter: Evidence-based guidelines for migraine headache (an evidence based review). Report of the Quality Standards Subcommittee of the American Academy of Neurology. Neurology 1994; 44:1353-1354.

9. Frishberg BM, Rosenberg JH, Matchar DB, et al. Evidence-based guideline in the primary care setting: neuroimaging in patients with nonacute headache. U.S. Headache Consortium Web site. Available at: www.aan.com.

10. Seifert $\mathbf{C L}$, Woeller $\mathbf{A}$, Valet $\mathbf{M}$, et al. Headaches and pineal cyst: a case control study. Headache 2008 Mar; 48(3):448-452.

11. Jordan JE, Ramirez GF, Bradley WG, Chen DY, Lightfoote JB, Song A. Economic and outcomes assessment of magnetic resonance 


\section{Original Article}

imaging in the evaluation of headache. J Natl Med Assoc 2000; 92:573-578.

12. Osborn RE, Alder DC, Mitchell CS. MR imaging of the brain in patients with migraine headaches. AJNR Am J Neuroradiol 1991; $12: 521-524$.
Chronic Headache and CT Scan

13. Kahn CE Jr, Sanders GD, Lyons EA et al. Computed tomography for non-traumatic headache: current utilization and cost effectiveness. Can Assoc Radiol J. 1993 Jun; 44(3):189-193.

Correspondence Address: Anish Subedee, Lecturer and Consultant Radiologist, Department of Radiology, Nobel medical College Teaching Hospital, Biratnagar-5, Nepal. E-mail: anish_subedee@yahoo.com 